\title{
The Introduction of Proof at the Secondary School in Cameroun: A First Approach trough the Study of Quadrilaterals and Triangles in the Textbook
}

\author{
Patrick Tchonang Youkap ${ }^{1 *}$, Judith Njomgang Ngansop ${ }^{1}$, Daniel Tieudjo ${ }^{2}$, Bettina Pedemonte ${ }^{3}$ \\ ${ }^{1}$ University of Yaounde 1, CAMEROON \\ ${ }^{2}$ University of Ngoundere, CAMEROON \\ ${ }^{3}$ San Jose University, USA \\ *CORRESPONDENCE: $\otimes$ patricktchonang@yahoo.fr
}

\begin{abstract}
The purpose of this article is to better understand how proof is introduced into the study of quadrilaterals and triangles in high school. To do this, we designed a grid to analyse mathematics textbooks in Cameroon francophone subsystems ( $7^{\text {th }}$ Grade and $8^{\text {th }}$ Grade). The Anthropological Theory of Didactics and the paradigms in geometry served as a theoretical framework for our analyses. The results of our analysis indicate that problems in the lessons section correspond to guided problems. These kinds of problems do not develop students' spirit of research and initiative. The authors of the textbook choose to teach the functioning of deductive reasoning in the $8^{\text {th }}$ Grade. They choose to introduce proof in the commented exercise section rather than lessons section. The learning problems proposed in the textbooks contain drawings wish have informative function and representative function. The preponderance of drawings with a representative function that have the same shape and name observed in textbooks can contribute to the construction of constant visual models in students' minds. This could lead to the superficial use of drawings in proof tasks.
\end{abstract}

Keywords: textbooks, proof, drawing, figure, triangle, quadrilateral

\section{INTRODUCTION}

In Cameroon as many countries of the world, proof is not an object of studies in geometry at the beginning of secondary school; more precisely, it is not part of the content of the modules recommended by the mathematics official syllabus. However, the deductive reasoning that is considered as proof (Duval, 1992) is part of the fundamental skills that the teacher has to develop to their students. It should be taught at the same time as the study of geometric objects. However, no guidelines are proposed to the teachers in the official syllabus on how this skill should be developed. No protocol is proposed for solving the tasks of proof and the form that the expression of a proof should take. It can be observed that the proof tasks have decreased considerably in the actual official syllabus of college (6 $6^{\text {th }}$ to $9^{\text {th }}$ Grade).

Stylianides (2009) thinks that mathematics textbooks can play an important role in students' opportunities to engage in reasoning and proving. According to him, many teachers' decisions about what tasks to implement in the classrooms, and how and when to implement them, are influenced by the textbooks they use. Mathematics textbooks used in Cameroon are designed to take into account curriculum recommendations. These textbooks are important resources for teachers and students in secondary schools. We think that an

Article History: Received 20 September $2019 \bullet$ Revised 30 April $2020 \bullet$ Accepted 22 June 2020

(C) 2020 by the authors; licensee Modestum. Open Access terms of the Creative Commons Attribution 4.0 International License (http://creativecommons.org/licenses/by/4.0/) apply. The license permits unrestricted use, distribution, and reproduction in any medium, on the condition that users give exact credit to the original author(s) and the source, provide a link to the Creative Commons license, and indicate if they made any changes. 
analysis of Cameroonian textbooks should reveal much about the approach taken by the authors of the textbook to introduce proof.

In some countries, such as France, Germany and Japan, proof is seen as something to be explicitly taught. Cabassut (2005) rapport that the introduction of proof in France and Germany are studies mostly in $8^{\text {th }}$ Grade, which is also the situation in Japan (Fujita \& Jones, 2014). In these countries, the official syllabus makes explicit what should be taught about proof as well as what textbooks contain chapters about proof (Cabassut, 2009; Fujita, Jones, \& Kunimune, 2009). We assume that, if what should be taught about proof which is absent in our official syllabus is not taken into account in the textbooks, they can have consequences on teachers' practice. This study intends to clarify the choice made by the authors of the textbooks about proof and predicts the repercussions of their choice on the students' learning.

As Stylianides (2009) points out, proof involves several other activities. Other factors need to be considered in studying how proof is taught, for example, research shows that illustrations of geometric objects influence student reasoning and proving in $8^{\text {th }}$ Grade (Coppé, Dorier, \& Moreau, 2005; Kerboeuf \& Houdebine, 2005; Njomgang \& Tchonang, 2018; Tanguay \& Geeraerts, 2012; Walter, 2001). Geometry at school is thought to develop before reasoning on the drawing which represents the figure and after reasoning based on the figure which is an abstract object, and to move from drawing to figure is not easy for them (Houdement \& Kuzniak, 2006). Some researchers (Duval, 1992; Tchonang, Njomgang, Tieudjo, \& Nchian, 2019) have shown that students have difficulty in producing acceptable proof. The work of Duval (1992) highlights the fact that students' difficulties in producing proof are due to misunderstanding of how deductive reasoning works. On the other hand, Tchonang et al. (2019) observes that students' difficulties come from a misuse of the drawing, but also from prototype phenomena. However, the origins of students' difficulties in producing acceptable proof are not sufficiently known, and there is a lack of knowledge about how textbooks influence students' initiation into deductive reasoning. We are not aware of much research that has been conducted on how proof is introduced in textbooks in African country. This research will provide information about how proof is introduced in some African countries, it will make it possible to establish a link between the students' difficulties and the phenomena observed in the textbook. In this article, we analyse a mathematical textbook by addressing the following question: what choices are made to introduce proof into the study of quadrilaterals and triangles at the beginning of secondary school in Cameroon?

To answer our research question, we organize this study as follows, we stat by present the relevant research on proof in mathematics education. After this we propose our theoretical framework, we situated ourselves within the framework of the Anthropological Theory of Didactics. Then we present the methodology we have chosen for our study. We developed a method of analysis that allowed us to examine not only the set of prescribed tasks, but also definition and theorem in the lessons. The mathematical textbook in our corpus is written in French, we transcribe the examples into English. We recognize that we are reporting on only one textbook, but our sample is from a major publisher and most popular textbook used in more than 20 Frenchspeaking Africa country. After methodology we present the results obtained and finally the discussions and conclusion.

\section{LITERATURE REVIEW}

Research on proof and proving had been addressed in mathematics education. The current literature review follows two axes: first, he summary of literature about reasoning and proof in mathematics textbooks; secondly, the summary of literature about proof and geometrical figure in secondary school.

With respect to textbook analysis research, Fujita et al. (2009) argue that the proof and proving activities in textbooks in Japan show geometry as a highly formal subject of study, which do not explain the differences between a formal proof and experimental verification. Fujita and Jones (2014) report that lessons are organized in blocks in Japanese mathematics textbooks which is the same in Cameroon. The lessons are introduced by the problem-solving activities contained in the blocks ${ }^{1}$ "activities of discovery". They lead students to make initial conjectures about the theorem studied in the lessons. Facts and theorems are present in the narrative blocks and are followed by application exercises. These results do not provide information on the types of problem and drawing proposed in the activist blocks, which we believe is relevant to understanding how proof is studied in textbooks. Some researchers such as Vinner (1983) carries out research on student cognitive structure about geometrical objects. He believes that, the way in which drawings are presented in

${ }^{1}$ A lesson section is constituted in blocks, and each block has a title, for example: "activities of discovery", "applications" and the other which is the narrative block where theorems are proposed. 
textbooks can lead students to construct incoherent mental images of the manipulated figure, and it is this mental image that is likely to be mobilized to accomplish proof task in problem-solving situation. In parallel, some researchers (Tanguay \& Geeraerts, 2012) have been interested in graphic illustrations of theorems and definitions in the study of geometric objects. They report that these illustrations can be used to make students understand the components of an argument in deductive reasoning. In particular this illustration has to separate the properties which correspond to the conditions with those which correspond to conclusion. These results are interesting, they indicate the elements that can be taken into account when one analysis how proof is introduced in a textbook, for example: the problem-solving activities, theorem formulations, figures and drawings. These results are useful to us in developing the analysis grid in the textbook for this study.

Some researcher has explored the cognitive dimension of proof and the links between proof and figure in secondary education. On cognitive aspect of proof at the beginning of secondary school, some researchers (Garuti, Boero, \& Lemut, 1998; Pedemonte, 2002) conducted research about continuity between argumentation and proof. They reported that open problem is relevant to make students discover proof. In particular, problems that lead students to initial produce the conjecture of a theorem before produce the proof of the conjecture are relevant to observe continuity between argumentation and proof. Walter (2001) was interested in the teaching of geometry at the beginning of secondary school, she reports that one of the students' difficulties in producing a mathematical proof derives from the superficial use of the drawing. Other researchers who focus on the link between drawing and proof reports that the use of freehand drawings in solving geometrical problems can have undesirable effects on students' knowledge construction (Coppé et al., 2005; Gobert, 2007). Furthermore, the use of prototype drawings in learning activities can contribute to the construction of a didactic obstacle in students' cognitive structure. The spatial position of the elements of a drawing can lead to inappropriate inferences. The results presented in this paragraph highlight some recommendations on learning proof, they concern the types of problems to be used in problem-solving activities as well as the types of drawings to be associated in problem statements. These results will be useful in discussing the results of this study.

Our contribution in this study is to enriching existing knowledge about learning proof by highlighting how proof is introduced within the study of figures in textbooks in Cameroon. The results of this study should highlight the choices made by the authors of the textbooks to introduce proof at secondary school in francophone subsystems in Cameroon. It should make it possible, on the one hand, to make hypotheses on the possible repercussions of the phenomena observed in the textbooks on students' cognitive structure about proof. On the other hand, we will be able to make suggestions for textbook authors about their choices in the treatment of the proof.

\section{THEORETICAL FRAMEWORK}

Our analyses of how proof is introduced through the studies of geometrical objects in the textbook follow an institutional approach. We intend to take into account the institutional choices and their repercussions (González-Martín, Giraldo, \& Souto, 2013). The institutional relation to an object is perceived as the set of possible interactions between institution I and object O (Chevallard \& Bosch, 2019). There should be some consistency between the students' personal relation to the object $\mathrm{O}$ and the institutional relation to the same object $\mathrm{O}$.

Anthropological theory attempts to better describe the choices made by an institution when organizing the teaching of an object, and the consequences of these choices on the meaning given to the object being taught, as well as on the learning achieved. From an Anthropological Theory of Didactics (ATD) perspective, all human activity consists of accomplishing a ' $\mathrm{t}$ ' task of a certain type ' $\mathrm{T}$ ' by means of a technique $\tau$ which is a way of


$h a w)$. The technique is justified by a technology $\theta$ which allows at the same time to think of it, even to produce it. This technology is in turn justifiable by a theory $\Theta$. These two elements constitute the knowledge bloc (theoretical bloc). Chevallard (2007) designed these four components $[\mathrm{T} / \tau / \theta / \Theta]$ as praxeology or praxeological organization. The praxeology is fundamental aspects of the anthropological model of mathematical activity which can be used to describe mathematical knowledge (González-Martín et al., 2013). For example, for the task of proof that two given straight lines are parallel, different techniques can be used depending on data and on how the data is provided in the statement (on the drawing or by text), data that can help to infer the conclusion. These different techniques can be explained by technologies (if two lines are perpendicular to the same line then they are parallel, etc.) that make sense in the theory of Euclidean geometry. 
In anthropological theory, some searcher characterizes the problem which is usually used in textbooks, they call them prescribed tasks. They are considered as a couple of statements and the institutional context of prescriptions (Chaachoua, 2010). The statement is a textual presentation of the mathematical problem, possibly adding indications or questions that partially or completely support the steps of the expected technique. The institutional context specifies the elements of the context in which the task is prescribed. The choice of a technique and its implementation by the student depends on the elements of this context. Chaachoua (2010) introduces the concept of mathematical task which he associates with the concept of prescribed tasks. According to him, it is a problem with no indication of the possible techniques expected, the formulation remains as neutral as possible with regard to technique. The prescribed task is constructed by the researcher in relation to the mathematical organization of the prescribed task without taking into account the didactic intentions of the institution. From the above, we distinguish two types of prescribed task: Those whose statement contains indications and intermediate tasks that support the expected technique which we call guided problems, and those which do not contain indications and no intermediate tasks that support the expected technique which we call mathematical task (open problem).

The anthropological theory allows us to describe the praxeological organizations contained in the prescribed tasks used to learn geometric objects in the textbook. They will allow us to describe the choices made in the textbooks to present technology into narrative blocks and their implication in initiation on deductive reasoning. They will allow us to predict the possible impact of phenomena in the textbook on the student's comprehension of proof.

\section{METHODOLOGY}

In this section, we present the methodology that we adopt to conduct this study. It is an explorative inductive search that follows a qualitative approach.

\section{Sampling}

We use the case of Cameroonian textbooks as an example that will allow us to raise and to discuss some questions which, we believe, have general parallels with the teaching of proof worldwide. In Cameroon secondary school is organised in two sections: French-speaker section and English speaker section. Frenchspeaker section is organised in two subsections:

- the first cycle ( $6^{\text {th }}$ Grade to $9^{\text {th }}$ Grade, age 11 to 15 years old), this cycle is organised in two sub-cycles. The sub-cycle of observation ( $6^{\text {th }}$ Grade to $7^{\text {th }}$ Grade, age 11 to 13 years old) and the sub-cycle of orientation ( $8^{\text {th }}$ Grade to $9^{\text {th }}$ Grade, age 13 to $15 y$ ears old);

- the second cycle (10 ${ }^{\text {th }}$ Grade to $12^{\text {th }}$ Grade, age 15 to 18$)$.

For our research, we use empirical data from a textbook used in institutions $8^{\text {th }}$ Grade (name of the textbook, CIAM4e) in more than 20 French-speaker country in Africa. In fact, the mathematics official syllabus prescribes to develop the skills related to the proof in this institution. This textbook is from a collection that was adopted in 1994 in 20 French-speaker African countries and are style used nowadays in many countries. The name of the collection is "Collection Inter Africaine de Mathématique", it was revised in 2008 and is still in use today; this collection is the most widespread in the national territory and is accessible to all segments of the population. All Teacher in Cameroon know this collection, some used it more than 10 years as a teacher the other as a student.

The textbook is organized in two main parts: a part called "numerical activity" and a part called "geometrical activity".

Table 1 shows that geometry occupies an important place in the teaching of mathematics in $8^{\text {th }}$ Grade institutions. More than 50\% of the chapters of our textbooks are devoted to it. One chapter of the textbook is organized into three sections. The first sections of the chapter contain lessons, follow by the section solved exercise and finally the section wish contain exercises. A lesson is organized into blocks, it can be: "Activities of discovery" (problem-solving activities), they are blocks which contained prescribed tasks, these tasks are used to make students learn the mathematics object; the narrative block, where technologies as theorem, properties and definition are presented. Finally, at the end of the section they are blocks "Applications" in which the prescribed tasks that the technique to accomplish the tasks is justified by the technologies study in the lessons of the section. The contents on quadrilaterals and triangles are studies in the first three chapters of the part "geometrical activity" of our textbook. The chapter on quadrilaterals and triangles constitutes the 
Table 1. Distribution of content in textbooks

\begin{tabular}{ccc}
\hline Numerical Activity & Lesson & Geometrical activity \\
\hline Chapter 1: Symmetry & 5 & Chapter 10: literal Calculi \\
\hline Chapter 2: Distance & 7 & Chapter 11: Rationales Numbers \\
\hline Chapter 3: Triangle & 10 & Chapter 12: Equations -inequation \\
\hline Chapter 4: Translation et vectors & 8 & Chapter 13: decimals Approximations \\
\hline Chapter 5: Projection and location & 5 & Chapter 14: problems solving \\
\hline Chapter 6: Center angle-regular polygon & 4 & Chapter 15: Statistics \\
\hline Chapter 7: Solid of space & 9 & \\
\hline Chapter 8: Straight lines and plane in space & 9 & \\
\hline Chapter 9: Number of pages: 120 & & Number of pages: 109 \\
\hline
\end{tabular}

Table 2. Distribution of prescribe tasks in the block: "activities of discovery"

\begin{tabular}{cc}
\hline Prescribed tasks & Percentage \\
\hline Proof tasks & $64.29 \%$ \\
\hline Empirical tasks & $35.71 \%$ \\
\hline
\end{tabular}

content of this study. These contents occupy a significant place in "geometrical activity" in our textbook: $21 \%$ of the lesson is devoted to them. We believe that in these institutions, the study of this figure can serve as a basis for the initiation of students to the practice of the proof.

\section{Data Analysis}

Our analysis of the institutional relationship to the proof in the textbooks are made in two phases (González-Martín et al., 2013): the first phase concerns the prescribed tasks proposed in the blocks "activities of discovery" and the prescribed tasks proposed in the solved exercise blocks. The second phase concerns the narrative block. In the first phase, we describe the types of prescribed tasks that are proposed in the lesson of our chapter, and then we describe the praxeological organizations related to the proof that is contained in these tasks. The drawings contained in the problem statements are analyzed in other to highlight their function in the problem-solving. The prescribed tasks in the section "solved exercise" are analyzed and the forms of proof proposed in the textbook are described. In the second phase, the technology (definition, theorem, method) that are proposed in narrative block of the lessons are analyzed, to highlight their formulation as well as their contribution to the study of deductive reasoning. The diagrams used in the textbooks to illustrate these technologies are analyzed to highlight the contributions they can have on the study of the component of an argument.

\section{RESULTS}

In this section, we present and analyse the collected data to provide answers to our research question. This presentation is organized into two sub-sections. The first sub-section deals with first phase of our data analysis and the second sub-section deals with the analysis of the narrative block and corresponds to the second phase.

\section{Phase 1}

\section{Prescribe tasks as learning problem in lesson}

The prescribes tasks are present in the blocks "activities of discovery". These prescribe tasks are problem which supposed to make students learn technology as definition and theorem on quadrilaterals and triangles. Table 2 presents the distribution of prescribed tasks according to the type of tasks of the mathematics tasks associate to the prescribed tasks in the lessons.

Table 2 shows two categories of prescribed tasks in the block "activity of discovery" of the lessons. The first category is constituted of prescribed tasks whose mathematics tasks is to prove an assertion, a property of a geometrical object. Those tasks are the predominant ones in the lessons of our chapters. One can suppose that textbook gives an important part of deductive reasoning in student activities. The second category is constituted of prescribed tasks which mathematics tasks need experience, for example: Search for a measure of the geometric object; construct a geometric object; recognize a geometrical object, discover a property, a theorem and produce a drawing of the geometrical object. These empirical tasks occupy a significant place in our lesson. They are suggesting different ostensible operations made on one or more figures in order to observe a property of a triangle or a quadrilateral. 


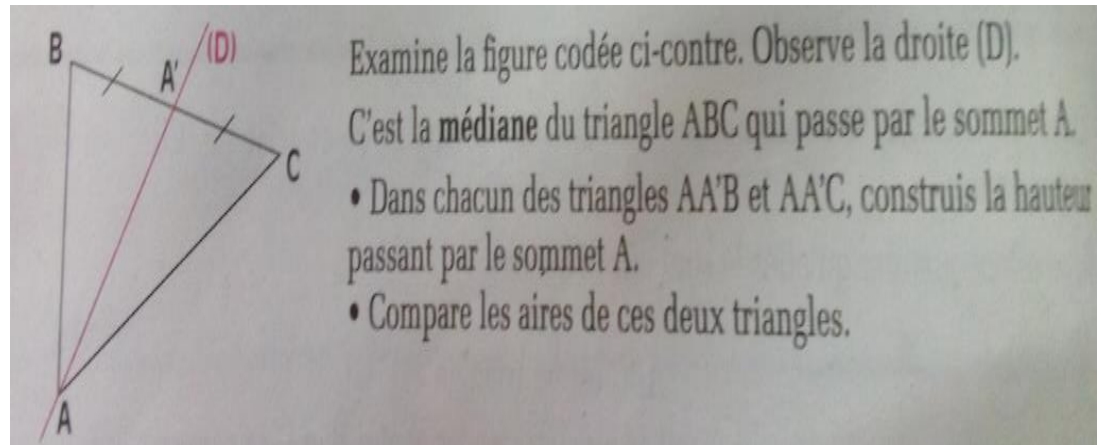

Figure 1. Proposed problems to discover a property, page 50 (Look at the figure opposite. Look to the straight line (D). It is the median of the triangle $A B C$ that passes through the vertex $A$. - In each of the triangles $A A$ 'B and $A A^{\prime} C$, draw the height passing through vertex $A$. - Compare the areas of these two triangles)

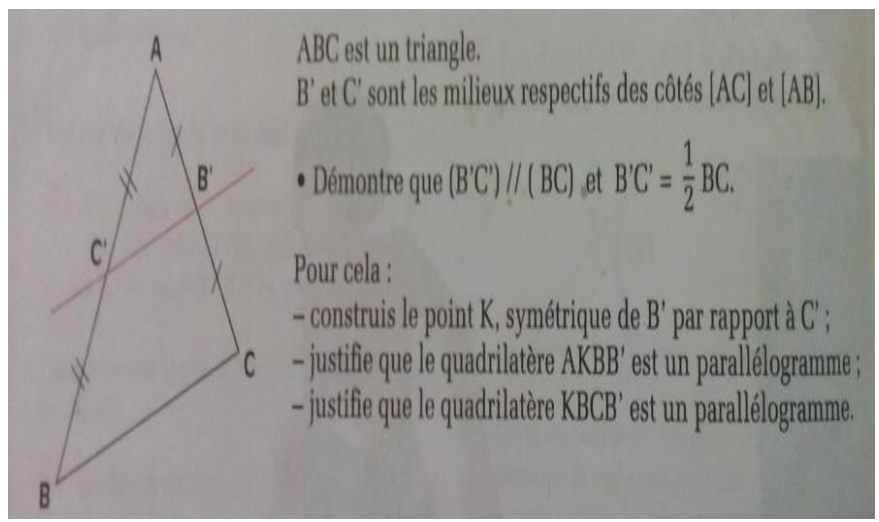

Figure 2. Proposed problem to prove a proposal, page 46 (Questions translate into English

$A B C$ is a triangle. $B^{\prime}$ and $C^{\prime}$ are the respective midpoints of the $[A C]$ and $[A B]$ sides. Prove that $\left(B^{\prime} C^{\prime}\right) / /(B C)$ and $B^{\prime} C^{\prime}=1 / 2 B C$. For this purpose: -Draw the point $K$, symmetrical from $B$ ' to $C$ '; -Justifies that the quadrilateral $A K B B^{\prime}$ is a parallelogram; -Justifies that the quadrilateral $K B C B$ ' is a parallelogram).

The prescribed tasks whose correspond to empirical tasks are problems which are divided in parcel tasks that support the entire technique that can be used to accomplish the mathematical task which is associate to these prescribed tasks. These are problems that provide assistance to the student in the form of an intermediate task. To solve these problems, students have to work on the drawing (interpretation, draw, etc.). Here is an example of a problem whose intention is to make students discover that the median divides a triangle into two triangles of the same area.

Two types of tasks can be identified in this problem that belong respectively to the kinds of tasks: draw and compare. These are tasks that need to work on the drawing. This problem is a guided problem, the technologies which justify the technique to accomplish the tasks are assumed to be known by the students because it is already learned. Furthermore, the median is presented to students on the drawing. One can fear that students may construct incoherent mental images on the median within a triangle. He can construct a personal representation of the median by interpreting is drawing, which is only an economic substitute for the figure (Vinner, 2002). The mathematical task that can be associated with this prescribed task is the following: "ABC is a triangle, draw a straight line (D) that divides the triangle into two triangles of the same area".

The prescribed tasks which mathematical tasks belong to proof tasks are designed to guide students to a particular technique. In these problems, the conjecture to be proof is explicitly give. One observes that there is a main task, and intermediate tasks that are the steps to solve the main task. Proof tasks are introduced by the action verb 'prove'. Furthermore, the intermediate tasks that constitute the technique to accomplish the proof task are sometimes introduced by the verb 'justify'. Here is an example of problems whose objective is to prove the property of the 'middles line in a triangle'.

Three types of tasks can be observed in the previous problem, one of the three types of tasks belong to the kind of task "draw" which belong to empirical geometry, and the other two belong to the kind of task "prove" which belong to deductive geometry. The use of two action verbs, namely "justify" and "prove", to prescribe 
Table 3. Distribution of the different proof tasks in the lessons

\begin{tabular}{cc}
\hline Types of tasks & Numbers \\
\hline Justify that two segments have the same length & 3 \\
\hline Justify that two lines are perpendicular & 5 \\
\hline Justify that a point is the middle of a segment & 2 \\
\hline Prove that two straight lines are parallel & 5 \\
\hline Justifying that a quadrilateral is a parallelogram & 4 \\
\hline Prove that a point is the middle of a segment & 2 \\
\hline Prove that a straight line is the perpendicular bisector of a triangle & 1 \\
\hline Prove that a point belongs to a straight line & 2 \\
\hline Prove that a straight line and a circle are tangent to a point & 1 \\
\hline Justify that a straight line is the axis of symmetry of an isosceles triangle. & \\
\hline Justify that two triangles have the same area & 1 \\
\hline
\end{tabular}

proof tasks seems to indicate that for the textbook these tasks are different. One can suppose that, in the textbook, the distinction between these two verbs lies in the number of deductive steps. The verb justify is used when it is only one deductive step. The mathematical proof is described in the textbook as follows: "To prove is to establish a succession of steps that, starting from the data, leads to the conclusion, each of these steps being justified by definitions, properties or formulas" (page,6). This prescribed task is a guided problem, the technologies that justify the techniques to accomplish the tasks belong to student's culture. The mathematical task associate to this task could be the follow: "ABC is a triangle, $\mathrm{B}$ ' and $\mathrm{C}$ ' are the respective middle of the sides $[\mathrm{AC}]$ and $[\mathrm{AB}]$. Prove that $\left(\mathrm{B}^{\prime} \mathrm{C}^{\prime}\right) / /(\mathrm{BC})$ and $\mathrm{B}^{\prime} \mathrm{C}^{\prime}=1 / 2 \mathrm{BC}$."

The types of tasks identified in the prescribed tasks of the lesson of our chapter belong to the following kinds of tasks: prove; Draw; calculate; compare; measure and discover. $40 \%$ of type of tasks contain in problem in the block "activities of discovery" belong to the kind of task, "prove". The following table gives these main types of tasks.

The technologies that justify the techniques which allow to accomplish the tasks of proof in the textbook are part of the institutional context of the prescription. These are statements that for some have been taught in previous classes and others are studies in the lessons that precede the lesson which these tasks are proposed. These tasks are at the level of the students, at this level, the figure is supposed to be the support of the reasoning. Drawings that represent these figures are contained in the problem statements; they have an influence on the students' argumentation (Tchonang et al., 2019).

To conclude, we observed that the textbooks give an important place to the practice of proof in the prescribed tasks in the lessons. However, these prescribed tasks correspond to guided problems in which the proof tasks are subdivided into intermediate tasks that are too detailed. These types of problems are not relevant to develop creativity and heuristic knowledge to the students. They may not understand the coherence between these intermediate tasks. It can also be observed in the prescribed tasks that the initial conjectures of the theorems are not left to the students. These prescribed tasks proposed in the lessons of our chapter do not correspond to those suggests to promote continuity between argumentation and proof (Pedemonte, 2002).

\section{Drawings in prescribed tasks of the lesson}

Two types of drawings are used in prescribed tasks in the lessons on quadrilaterals and triangles. The first type is the drawing with the representative function in problem-solving situation. That means the drawing represents a part or all the property of the figure describes in the text of the problem statement. Most of the drawings 61,3\% associated with the problems have a representative function. In Figure 1 and Figure 2 of this article, we can see drawings which have a representative function. The presence of these drawings in the problems are not really necessary. With regard to the institutional context in which the task is prescribed, the technique and the technology that justify the technique to draw a triangle and the middle of a segment is supposed to be part of the students' culture. This kind of drawing does not provide additional information to solve the problem. The red colour is used to represent some configurations of the drawings. That may be indications that help to solve the prescribed task by increasing the perceptible apprehension of one configuration of the drawing.

The second type of drawing has informative function in problem-solving situation. It means the drawing contain information that is not described in text form in the problem statement. These information's are essentials to solve the prescribed tasks. $38.46 \%$ of drawings have informative function in problem-solving 


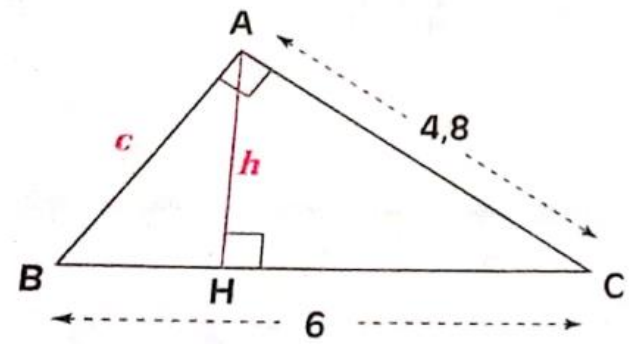

L'unité de longueur est le cm. Examine la figure codée ci-contre.

- Calcule $c$.

- Calcule l'aire du triangle ABC.

- Déduis-en $h$.

Figure 3. Drawing with an informative function page 56 (Questions translate into English

The unit of length is cm. Examine the coded figure opposite. Calculates c. Calculates the area of the triangle ABC. Deduct from $h$.)

situation. Students are explicitly asked to use the drawing contained in the problem statement. The red colour is also used on the drawing to represent some information on the drawing. The following example illustrates drawings that have an informative function in the textbook.

The drawing of the previous problem is essential to solve the prescribed task because it provides the necessary information to solve it (the measure of the side, the measure of angles the nature of triangles). Moreover, the prescribed tasks in which one can observe these drawings belong to the category of guided problems. It can be assumed that the work of interpreting the drawing is one of the skills that the textbooks want to develop in this problem.

The majority (70\%) of the drawings have the same form, orientation and some of them the same name (the $\mathrm{ABC}$ triangle). These drawings can lead students to construct prototype figures by memorizing the shape and orientation of the drawings. According to Vinner (1983), based on these drawings, students can construct a visual model of the figure, and then during the proof task they can produce informal proof base on this mental image evoked in their mine.

To conclude, the textbook proposes drawings with prescribed tasks in the lessons. In some cases, drawing has informative function, in other cases, the drawing has representative function in solving-problem situation. One can be assumed that the choices of proposing drawings that have a representative function in problemsolving are help to students. Indeed, this can lead them to no longer draw figures and save time. However, drawings that have the same shape and orientation can give rise to prototype phenomena. This may lead them to produce informal proof by using these mental image (Vinner, 1983) rather than the property of the figure to produce their argument (Tchonang et al., 2019).

\section{The solved exercise section of the chapter}

A section in the chapters of this study is reserved for solved exercises. These are prescribed task that contain a single task. The types of tasks proposed there belong to the kind of task: "prove and draw". All the prescribed task in this section except one doesn't contains a drawing in its statement. This drawing has an informative function in problem-solving. These problems are complex problems whose solution is not a simple application of a theorem or property. However, the technologies that justify the techniques to accomplish these tasks are part of the institutional context of prescriptions. Indeed, they have been studied in the lessons in this chapter but also in the lessons prior to the chapter. The problem that follows is one of the problems proposed in the textbook and whose type of task belongs to the kind of task "prove" on page 57.

$A B C$ is a triangle, the height passing through $A$ cuts the line $(B C)$ in $K . D$ is a point on the line $(A K)$ not belonging to the half-line [KA). The line parallel to the line (AB) passing through $D$ cuts the line (BC) into $E$. The line perpendicular to the line $(C D)$ passing through $E$ cuts the line $(A K)$ into E. Prove that the straight lines (BH) and (AC) are perpendicular.

(it can be justified in advance that point $C$ is the orthocentre of the DEH triangle)

The type of task of this prescribed task is: prove that two straight lines are perpendicular. The technology that justifies the technique needed to solve this task is part of the institutional context of prescriptions. At the end an indication is given to the students about the solving technique. This makes this problem a guided problem.

A protocol of resolution of those problems is proposed in the textbook, this protocol is divided into three steps: 


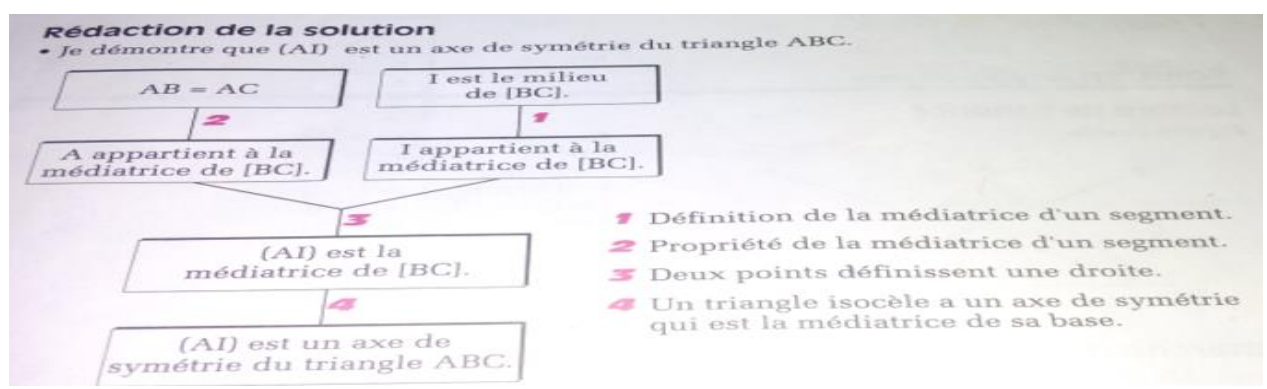

Figure 4. Deduction diagram in the textbook (Task: I prove that (AI) is an axis of symmetry of the triangle $A B C .1)$ definition of the mediator of a segment; 2) property of the mediator of a segment; 3) two points define a segment; 4) an isosceles triangle has an axis of symmetry which is the mediatrix of its base)

Table 4. Extract of a two-column proof

\begin{tabular}{cc}
\hline Argument & Justifications \\
\hline (EA) $\perp(\mathbf{D H})$ and $(\mathbf{C D}) \perp($ EH). & Definition of a hauteur of a triangle \\
Then (EA) and (CD) are the height of DEH triangle. & Definition of the orthocentre of a triangle. \\
Their intersect point is orthocentre of this triangle &
\end{tabular}

- Reading the statement: this is a phase where the student reads the statement, appropriates it, constructs the drawing, identifies the information given in the problem statement, identifies the conclusion that is the proposal to be proved;

- Seeks a strategy: this is the phase which the student seeks the appropriate proof technique;

- Writing the solution: this is the phase where the student writes the proof.

Tree forms of presentation of proof have been identified in the textbook: proof in the form of a deductive diagram; two-column proof and three-column proof.

In the proof which is present as a deductive diagram. An argument consists of the boxes that constitute the data followed by a number and a box that constitutes the conclusion. The conclusions of the previous arguments are recycled (Duval, 1992) into data for the next argument. The warrant which are definition, property and theorem are coded in the diagram by numbers. These numbers are interpreted near these diagrams. The example which follow illustrates this form of proof.

In this diagram, an argument consists of consecutive boxes separated by a number. For example, data: $\mathrm{AB}=\mathrm{AC}$; warrant 2; conclusion $\mathrm{A}$ belongs to the mediator of $[\mathrm{BC}]$. The number is a code that represents the warrant of the argument. In this example, there are four arguments. The four technologies that serve as a warrant are explained near the diagram. By this choice, one can assume that, the textbook helps students to understand the components of an argument as well as the transitions between arguments.

In two-column proof, the first column shows the arguments that are statements consisting of the data and the conclusion, and the second column shows the technologies (theorems and definitions) that serve as a warrant of these arguments.

In the previous example, we can see in the left column the arguments and in the right column the warrant that legitimizes the transition from the data to the conclusion. The recycling of data is implicit in these arguments. Indeed, the conclusions of the previous arguments that constitute the data for the following argument are not explicitly repeated. It may be assumed that for textbook, recycling is part of the students' culture. From our point of view, this choice may have undesirable consequences in the acquisition of transitions between arguments. Students' may think that, these arguments are independent, which is not true.

In the three-column proof, the data is contained in the first column, the conclusions are contained in the second column and the warrant are contained in the third column. Some terms are introduced to formulate the components of the argument: "I know that" which introduces the data, " then" which announces the conclusion and "because" which announces the warrant. The example below illustrates this three-column proof in the textbook.

The form of proof present in the textbook highlights the components of an argument, namely data, conclusion and warrant. Textbooks allow students and teachers to choose the form of proof they deem relevant. Presumably, this choice can help students to acquire the functioning of the proof. The two-column proof are 


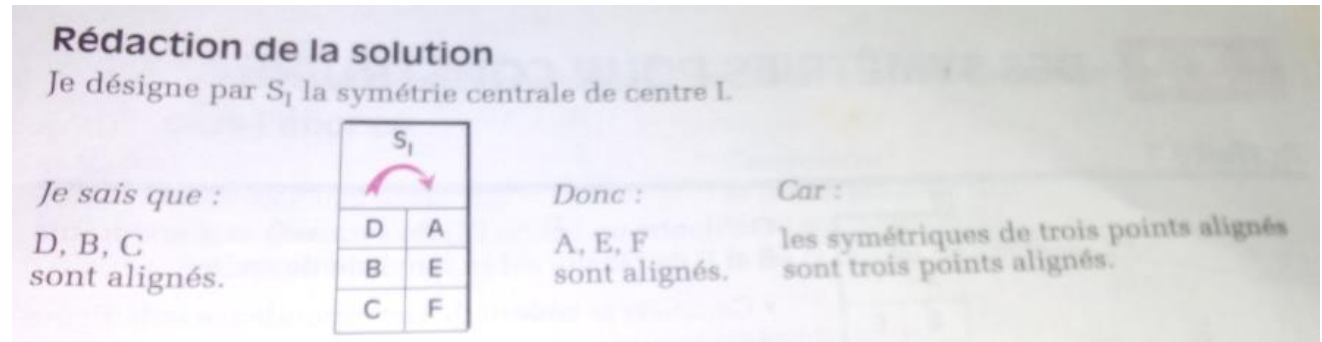

Figure 5. Three-column proof in the textbook (I designate by S1 the symmetry of centre I. I know that D, B and $C$ are aligned; $S 1(D)=A ; S 1(B)=E ; S 1(C)=F$ so $A, E, F$ are aligned because the symmetries of three aligned points are aligned)

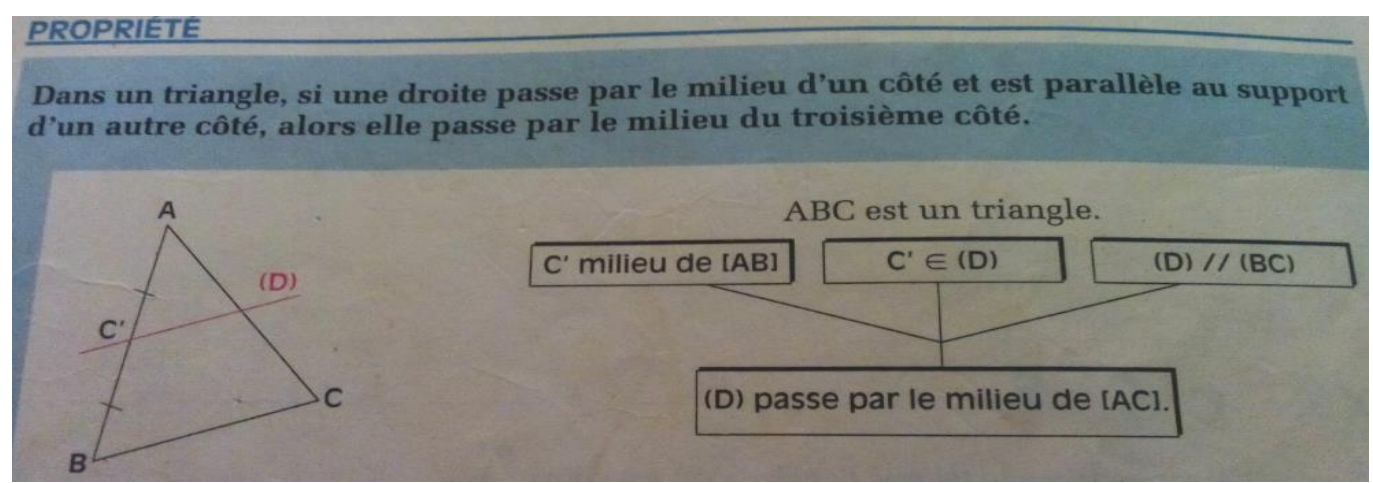

Figure 6. Illustration of a theorem from a drawing and an inference diagram (Property. In a triangle, if a straight line passes through the middle of one side and is parallel to the support of another side, then it passes through the middle of the third side)

used in several countries around the world (Japan, United States, etc.), this form of proof is adjudged relevant by these countries to help students understand how mathematical proof works.

\section{Phase 2}

In this section we analyze the narrative blocks in which the technology (definition, theorem, formula) studied in the lessons are presented. Two form of presentation are identified: the statement of the technology and schemas which illustrate them.

They are many definitions in the narrative blocks in the chapters of our study. These definitions are provided axiomatically. Indeed, they are not introduced by prescribed tasks which allow students to construction definition. Because of that, this may favor the construction of students' conceptions on the figure that are in contradiction with the theory (Vinner, 1983). Furthermore, it may have repercussions on students' arguments in their proof.

Two forms of formulations have been retained to present theorems in geometry chapter of the textbook: the linear form $56 \%$ and the conditional form $46 \%$. The linear form is of the type, "A is B", for example, "a triangle that has an axis of symmetry is an isosceles triangle". The conditional form uses the terms "if" and "then", the "if" introduces the conditions and the "then" conclusion. For example, "if a triangle has an axis of symmetry then it is an isosceles triangle". In this formulation, one can read a condition (having an axis of symmetry) that a triangle must satisfy in order to infer the conclusion (the triangle is isosceles). This formulation corresponds to the hypothetical syllogism, and seems relevant to acquire the conditions for applying this theorem (Gousseau-Coutat, 2006).

The analysis of the illustrations of the theorems and definitions allowed us to identify two types of schemas. The first type of schema consists of a triangle on which we can read the properties of the figure, this triangle is associated with a deductive diagram consisting of the data and the conclusion. This type of diagram is used to illustrate theorems presented in conditional form.

The above example shows a triangle $\mathrm{ABC}$, a parallel line to the side (BC) that runs through the middle of one side $[\mathrm{AB}]$. The associated diagram is constituted by three box that contain the data and one box that contains the conclusion. The data is congruent to the information's in the condition part of the theorem and 


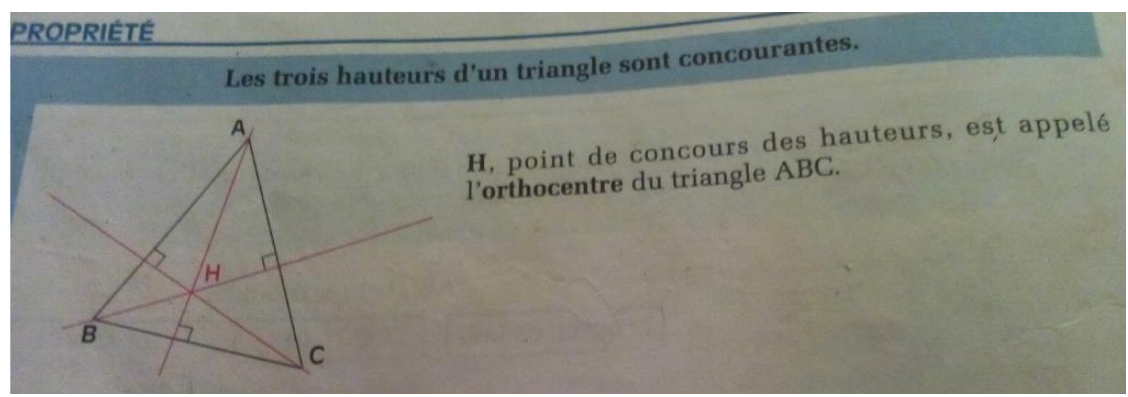

Figure 7. Illustration consisting of a triangle associated with an interpretation (Property: the three heights in a triangle are intersected at one point. Interpretation of the drawing: $H$, the point of intersection of the heights, is called the orthocenter of the triangle $A B C$ )

the conclusion is congruent to the conclusion part of the theorem. The acquisition of knowledge of applying the theorem in a proof can be promoted by this type of illustration. Indeed, the diagram presents the components of the argument separately as well as the conditions of the application of the theorem. The diagram shows that there is no confusion on the theorem, it is the direct property that is illustrated and not its reciprocal.

The second type of diagram consists of a drawing on which the properties of the figure can be read. Near these drawings sometimes interpretations of the properties represented on the drawing is proposed. This type of illustration is used to represent theorems and definitions that are proposed in linear form.

The diagram in this example shows a drawing of a triangle $\mathrm{ABC}$ in which the three heights have been represented in red. The interpretation of this triangle reveals that the point $\mathrm{H}$ is the orthocenter of the triangle. This type of illustration could be used to acquire the skills to interpret a drawing. This skill is necessary when in solving-problem situation, students want to choose relevant information on the drawing which can be used in an argument.

To conclude, this section allows us to see that the narrative blocks of the lessons in our chapter also favor the acquisition of deductive reasoning. Some relevant elements to produce proof are proposed, namely, the deductive diagrams associated with coded triangles, as well as triangles associated with their interpretation. The conditions of the application of a theorem and the judicious choice of information read on the drawing are essential to produce acceptable proof of the institution where this textbook is used. However, proposing definitions axiomatically can be a handicap in the practice of proof (Tchonang et al., 2019). This is because what is evoked in the cognitive structure of the student in a problem-solving situation is a set of properties and mental image on the manipulated figure. It is therefore imperative that the properties contained in the definition are consistent with the mental representations of the student on that figure. It can be assumed that for the definition to be used by students as a warrant in an argument, they must have been involved in its construction.

\section{DISCUSSIONS}

We can begin by reminding our research objective, which was to understand the choices made in the textbook to introduce proof through the study of quadrilaterals and triangles at the beginning of secondary school in Cameroon.

Some aspects fond in the textbook are relevant to introduce deductive reasoning to students.

Firstly, in view of the results of this study, the textbooks seem to have chosen to develop the practice of proof in the blocks "activities of discovery" of the lessons on quadrilaterals and triangles. Indeed, the kinds of tasks "prove" occupy a significant place in prescribed tasks. These results agree with those of Fujita and Jones (2014) and Fujita, Jones and Kunimune (2009) who make the same observation in Japanese textbooks.

Furthermore, our result indicates that the textbook suggests different forms of presentation of proof. Three forms of proof are identified in the geometry chapters: deductive diagram, three-column proof and two-column proof. These different forms of proof proposed in the textbook highlight the components of an argument. Some countries have opted to use a single form of proof, the two-column proof is in the form of proof adopted by the United States of America to enable students to understand how proof function (Miyakawa, 2012). These forms of proof are all relevant to introducing students to the functioning of deductive reasoning. 
Lastly, the narrative blocks in which technology (theorems and definitions) study in the lesson is proposed are also used to initiate students to deductive reasoning. The formulation of the technology as well as the illustrations chosen in the textbook promote the learning of deductive reasoning. Indeed, the deductive diagrams that are used allow to understand how an argument works. In addition, drawings and their interpretation can help students to avoid abusive interpretations of drawings in problem-solving situations. At this point, the suggestions of Tanguay and Geeraerts (2012) to use two drawings to illustrate a theorem in conditional form does not correspond to those proposed in the textbook. However, the presence of theorems and their graphical illustration in the narrative blocks of the textbook seem relevant to help students to acquire deductive reasoning.

Some phenomena observed in the textbook are not relevant to develop students' competence in deductive reasoning. Firstly, the prescribed tasks in the lessons are guided problems, the mathematical tasks which are associated are decomposed into intermediate tasks that serve as indications of the technique to accomplish them. In these prescribed tasks the first conjecture of the theorem is not left to the student. These results are not consistent with the results of Pedemonte (2002), Mariotti (2000) and Fujita and Jones (2014). These authors suggest that problems (in particular problem-situations) which conducted the student to make first conjecture of a theorem and after produce the proof of this conjecture are relevant to discover proof. The choice made in the textbook to offer help by dividing a proof tasks into tasks that are too detailed can turns the student into a skilled worker having to perform only fragmented tasks that they don't understand the coherence. These prescribed tasks may not develop the student's spirit of initiative, inventiveness and creativity (Arsac, Germain, \& Mante, 1988). Furthermore, it may hinder students' acquisition of the heuristic skills needed to solve proof tasks that require several steps to be solved.

In parallel, the textbook has chosen to associate drawings in the statements of prescribed tasks in the lessons. In particular, those in the lessons on quadrilaterals and triangles. These drawings contain indications in the form of color, some have the same shape, the same orientation. Previous research (Coppé et al., 2005; Gobert, 2007) suggests using a variety of drawings, especially to vary their forms and orientation to prevent prototype phenomenon which affect students argumentation. This prototype phenomenon identify could influence arguments that students use to produce informal proof (Tchonang et al., 2019).

\section{CONCLUSION}

The objective of this article was to highlight the choices made in the textbook to introduce proof through the study of quadrilaterals and triangles in secondary school in Cameroon. In order to provide some answers to our problem, we opted for a descriptive qualitative study.

In relation to our research question, we can say that all the sections of the chapter are used in the textbooks to introduce proof. The prescribe tasks use as problem-solving activity in the lessons are used to develop the practice of proof among students. However, the fact that students only have to solve fragmented tasks of proof can affect their understanding of this practical knowledge. Furthermore, students may not understand the coherence between the tasks they are asked to perform. Our result also highlights the fact that the first conjectures of the theorems are not left to the students; this may not favor the cognitive continuity between the argumentation that emerges during the construction of the conjecture and it proof. The narrative blocks where technology that justify a technique to accomplish a proof task is present are also used to introduce students to deductive reasoning. The formulation of theorems and their illustration can promote an understanding of the components of an argument as well as the conditions of their application as a warrant in an argument. We can also say that the sections reserved for solved exercises are used to promote understanding of how deductive reasoning works. The forms of proof that are proposed can promote the understanding of the components of an argument and the transitions between arguments.

We are conscious that, this study has limits, at first our corpus contains only one textbook, so the results cannot be generalized. Moreover, we think that these results would be richer if the number of textbooks became important. Finally, we think that the analysis of textbooks in the first cycle of secondary school could give more information about how proof is introduced in textbooks. However, the results of this study allow us to highlight the choices made in the textbook to introduce proof. They allow us to make hypotheses for a largescale study. It also allows us to make hypotheses about the origins of the students' difficulties in solving a proof task that requires several steps. We are planning to analyze a significant number of textbooks to complement our results on introduction of proof in textbooks. We also intend to analyze how teaching practices are developed, based on the assumption that teachers are impacted by the choice made in textbooks. 


\section{Disclosure statement}

No potential conflict of interest was reported by the authors.

\section{Notes on contributors}

Patrick Tchonang Youkap - University of Yaounde 1, Cameroon.

Judith Njomgang Ngansop - University of Yaounde 1, Cameroon.

Daniel Tieudjo - University of Ngoundere, Cameroon.

Bettina Pedemonte - San Jose University, USA.

\section{REFERENCES}

Arsac, G., Germain, G., \& Mante, M. (1988). Problème ouvert et situation-problème [Open problem and problem-situation]. IREM de Lyon.

Cabassut, R. (2005). Démonstration, raisonnement et validation dans l'enseignement secondaire des mathématiques en France et en Allemagne [Proof, Reasoning and Validation in Mathematics Secondary Schools in France and Germany] (Doctoral Dissertation). Université Paris Diderot, Paris VII. Retrieved from https://tel.archives-ouvertes.fr/tel-00009716/document

Cabassut, R. (2009). The double transposition in proving. In F.-L. Lin, F.-J. Hsieh, G. Hanna, \& M. de Villiers (Eds.), Proceedings of the ICMI Study 19 conference: Proof and Proving in Mathematics Education (Vol. 1, pp. 124-129). Taipei: National Taiwan Normal University.

Chaachoua, H. (2010). La praxéologie comme modèle didactique pour la problématique EIAH. Etude de cas: la modélisation des connaissances des élèves [The praxeology as didactic model for the EIAH problem] (HDR dissertation). Université de Grenoble. Retrieved from https://tel.archives-ouvertes.fr/tel00922383

Chevallard, Y. (2007). Readjusting didactics to a changing epistemiology. European Educational Research Journal, 6(2), 131-134. https://doi.org/10.2304/eerj.2007.6.2.131

Chevallard, Y., \& Bosch, M. (2019). Anthropological Theory of the Didactic (ATD). In S. Lerman (ed.), Encyclopedia of Mathematics Education, (pp. 1-8). Springer Nature Switzerland AG. https://doi.org/10.1007/978-3-319-77487-9_100034-1

Coppé, S., Dorier, J.-L., \& Moreau, V. (2005). Différents types de dessins dans les activités d'argumentation en classe de 5 ème[Different types of drawings in 7th Grade argumentation activities] Petit X, (68), 8-37.

Duval, R. (1992). Argumenter, démontrer, expliquer: continuité ou rupture cognitive[Arguing, proving, explaining: Cognitive continuity or Guap], Petit X, 31, 37-61.

Fujita, T., \& Jones, K. (2014). Reasoning-and-proving in geometry in school mathematics textbooks in Japan. International Journal of Educational Research, 64, 81-91. https://doi.org/10.1016/j.ijer.2013.09.014

Fujita, T., Jones, K., \& Kunimune, S. (2009). The design of textbooks and their influence on students' understanding of 'proof' in lower secondary school. In F.-L. Lin, F.-J. Hsieh, G. Hanna, \& M. de Villiers (Eds), Proceedings of the ICMI Study 19 conference: Proof and Proving in Mathematics Education (Vol. 1, pp. 172-177). Taipei, Taiwan: National Taiwan Normal University.

Garuti, R., Boero, P., \& Lemut, E. (1998). Cognitive unity of theorems and difficulty of proof. In A. Olivier and K. Newstead (Eds.), Proceedings of the 22th PME International Conference, (Vol. 2, pp. 345-352). Stellenbosch, South Africa.

Gobert, S. (2007). Conditions nécessaires à l'usage des dessins en géométrie déductive [Necessary conditions to use drawings in deductive geometry], Petit X, 47, 34-59.

González-Martín, A. S., Giraldo, V., \& Souto, A. M. (2013). The introduction of real numbers in secondary education: An institutional analysis of textbooks. Research in Mathematics Education, 15(3), 230-248. https://doi.org/10.1080/14794802.2013.803778 
Gousseau-Coutat, S. (2006). Intégration de la géométrie dynamique dans l'enseignement de la géométrie pour favoriser la liaison école primaire collège: une ingénierie didactique au collège sur la notion de propriété [Integration of dynamic geometry in the teaching of geometry to promote the link between primary school and secondary school: didactic engineering in secondary school on the notion of property] (Doctoral Dissertation). Université Joseph Fourier, Grenoble. Retrieved from https://tel.archives-ouvertes.fr/tel$00110052 /$

Houdement, C., \& Kuzniak, A. (2006). Paradigmes géométriques et enseignement de la géométrie [Geometric Paradigms and Geometry Teaching]. Annales de didactique et de sciences cognitives, 11, 175-193.

Kerboeuf, M.-P., \& Houdebine, J. (2005). Les figures-cles: une idee pour l'apprentissage de la demonstration en 4 [Key-figures: an idea for learning how to prove it in 8th Grade.], Repère IREM, 59, 83-103.

Mariotti, M. A. (2000). Introduction to proof: The mediation of a dynamic software environment. Educational Studies in Mathematics, 44(1), 25-53. https://doi.org/10.1023/A:1012733122556

Miyakawa, T. (2012). Proof in geometry: A comparative analysis of French and Japanese textbooks. In T. Y. Tso (Ed.). Proceedings of the 36th Conference of the International Group for the Psychology of Mathematics Education (Vol. 3, pp. 225-232). Taipei, Taiwan.

Njomgang Ngansop, J., \& Tchonang Youkap, P. (2018). La ligne droite, un objet d'étude au début du secondaire: une analyse institutionnelle des manuels [The straight line, a subject of study at the beginning of secondary school: an institutional analysis of textbooks]. Revue de Mathématiques Pour l'Ecole, 230, 23-29.

Pedemonte, B. (2002). Étude didactique et cognitive des rapports entre argumentation et démonstration dans l'apprentissage des mathématiques [A didactic and cognitive study of the relationship between argumentation and proof in the learning of mathematics.] (Doctoral Dissertation). Université JosephFourier-Grenoble I. Retrieved from https://tel.archives-ouvertes.fr/tel-00004579/

Stylianides, G. J. (2009). Reasoning-and-proving in school mathematics textbooks. Mathematical Thinking and Learning, 11, 258-288. https://doi.org/10.1080/10986060903253954

Tanguay, D., \& Geeraerts, L. (2012). D’une géométrie du perceptible à une géométrie déductive : à la recherche du paradigme manquant [From a perceptible geometry to a deductive geometry: in search of the missing paradigm], Petit $X, 88,5-24$.

Tchonang Youkap, P., Njomgang Ngansop, J., Tieudjo, D., \& Nchia Ntam, L. (2019). Influence of Drawing and Figures on Secondary School Students' Argumentation and Proof: An Investigation on Parallelogram. Acta Didactica Napocensia, 12(2), 133-144. https://doi.org/10.24193/adn.12.2.10

Vinner, S. (1983). Concept definition, concept image and the notion of function. International Journal of Mathematical Education in Science and Technology, 14(3), 293-305. https://doi.org/10.1080/0020739830140305

Vinner, S. (2002). The role of definitions in the teaching and learning of mathematics. In: Tall D. (eds) Advanced Mathematical Thinking. Mathematics education library, V11. Springer, Dordrecht. https://doi.org/10.1007/0-306-47203-1_5

Walter, A. (2001). Quelle géométrie pour l'enseignement en collège? [wath geometry for the teaching in secondery school], Petit $X, 54,31-49$. 